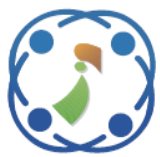

\title{
New Workflow for Marine Fish Classification Based on Combination Features and CLAHE Enhancement Technique
}

\author{
Ricardus Anggi Pramunendar ${ }^{1 *} \quad$ Dwi Puji Prabowo ${ }^{1} \quad$ Dewi Pergiwati $^{1}$ \\ Yuslena Sari $^{2} \quad$ Pulung Nurtantio Andono $^{1} \quad$ Moch Arief Soeleman $^{1}$ \\ ${ }^{1}$ Department of Informatics Engineering, Faculty of Computer Science, \\ Universitas Dian Nuswantoro, Semarang 50131, Indonesia \\ ${ }^{2}$ Department of Technology Information, Universitas Lambung Mangkurat, Banjarmasin 70123, Indonesia \\ * Corresponding author: ricardus.anggi@dsn.dinus.ac.id
}

\begin{abstract}
Automatic identification of fish species is very complex and challenging because of the low quality of the marine environment. Thus, the identification of fish species using computer vision technology is disrupted. However, various researchers only focus on determining the best fish identification method without considering the quality of the data used. Therefore, this study presented a new workflow in identifying fish species. A combination of feature extraction methods and a backpropagation neural network (BPNN) method was used, which was based on image quality improvement techniques using contrast limited adaptive histogram equalization (CLAHE) with adaptive threshold by fuzzy c-means. This study compared the results of fish identification on the original data and image data that were enhanced using several classifications of machine learning. The results show that data with improved quality of the images will improve accuracy for fish species identification and improvement using the proposed method of $3.56 \%$. This could support the reduction of invasive fish populations through automated fish identification systems in unrestricted natural environments based on computer vision technology.
\end{abstract}

Keywords: Image enhancement, Fish identification, NCACC, GLCM, Neural network.

\section{Introduction}

Threats to natural resources are growing, which makes efforts to protect aquatic biota populations are very challenging for many countries. World Bank data shows that there is an increase in the total number of endangered fish. In 2018, the total data on endangered fish reached 8,233 species [1].

The increasing number of extinct fish species is caused by growing environmental pressures on human needs so that a large number of fish species migrate and ultimately disrupt native fish populations. Efforts to overcome damage to native fish populations have been made by reducing invasive fish populations through traditional fishing techniques. However, these fishing techniques capture not only invasive fish species but also native species. Hence, a manual process is still needed to sort the catch, which consumes energy and takes time.
Besides, not all types of fish biota can be identified manually using human eyes [2]. Manual identification by humans is susceptible to unexpected things that affect the results of classification. Therefore, automatic classification based on artificial intelligence technology has a significant impact on biologists, the government, fishermen, and the community.

Nowadays, the automatic classification technique for objects in the marine ecosystem uses computer vision technology [3]. This technology has been implemented in various sectors. In the aquaculture sector, a back-propagation neural network (BPNN) method is used to classify marine fish species [1]. This technology has been widely used in recognizing underwater objects from image data that can be obtained using sensors. However, this technology depends on the environment and the object being 
observed [4]. Accurate observation can be achieved if the image source is in a noise-free environment [5].

The characteristics of the data are influenced by its origin, which means that data coming from underwater is influenced by various aspects of water. In this case, water characteristics differ significantly compared to air characteristics. However, water characteristics are influenced by its depth [1]. The deeper the water, the lower the color quality, brightness, contrast, and visibility. Some of these problems have been solved using the dark channel prior (DCP) technique proposed by CarlevarisBianco et al. [6], which was subsequently continued by Wen et al. [7] and updated by Kaur et al. [8], Galdran et al. [9], Gu et al. [3] and Pramunendar et al. [1]. However, previous works on improving image quality could not explain its effect on the performance of computer vision-based classifications for objects that are affected by underwater environmental problems.

To overcome this research gap, this study proposed a new workflow for identifying fish species. The workflow consists of extracting gray-level cooccurrence matrix (GLCM) features into the BPNN classification method with the contrast-limited adaptive histogram equalization (CLAHE) with a threshold as an image enhancement technique. The reliability of the CLAHE technique on improving image quality has been investigated by Pujiono et al. [10]. The reliability of the combination of the CLAHE method and a feature extraction method to be tested using the classification method developed by Pramunendar et al. [5]. However, the research [5] has not been explicitly analyzed the use of parameters in CLAHE when combined with the feature extraction method and tested using the classification method. Therefore, this study compared the distribution parameters and determined the value of the clip limit parameter on the image enhancement technique using CLAHE and compared with other image enhancement techniques such as dark channel prior (DCP), automatic level (AL), automatic white balance (AWB), and gamma correction (GC). Based on previous studies, GLCM can transform fish images into relevant fish features based on textures, and BPNN shows the best performance in previous studies [1].

The contribution of this study was validated by comparing the results of image enhancement based on classification methods to (1) show the effect of image enhancement on classification performance; (2) analyze the relationship between image quality and image classification performance; (3) determine the parameters needed to achieve the best performance results in the classification model, and
(4) present the best classification model results to recognize images of underwater fish.

The contents of this paper are organized as follows. In Section 2, previous research on this research is highlighted. Section 3 presents the proposed model. Section 4 explains the design of the experiment. Next, Section 5 explains the results of the experiment and discussion. Finally, the conclusions are presented in Section 6.

\section{Previous studies}

Marine and fishery biologists have worked for years to improve the accuracy of identifying marine fish species. However, almost all of them only consider improving image quality or classification, such as Lee et al. [11], Nery et al. [12], White et al. [13], Rova et al. [14], Larsen et al. [15], Khotimah et al. [23], and Iswari et al. [16].

Identification of marine fish species conducted by Lee et al. [11] identified 22 fish species based on contours and shapes. Nery et al. [12] and White et al. [13] used several fish size, shape, color, and texture features to identify several fish species. Nery et al. [12] used the Bayesian classification method to identify fish species, while White et al. [13] used the discriminant analysis method. Rova et al. [14] used the SVM method based on a linear kernel to identify the shape and texture features of four different fish species and produced an accuracy of $90 \%$. Larsen et al. [15] identified three fish species using the linear regression method and obtained an accuracy of $76 \%$. Khotimah et al. [17] identified tuna species using the Decision Tree (DT) method based on color, shape, and texture features with an accuracy of $88 \%$. Iswari et al. [16] used the K-Nearest Neighbor (KNN) method based on the color of the fish species to identify features with an accuracy of $91.36 \%$.

Among these studies, there are only a few studies that test the ability to improve image quality to be applied to identification methods, such as $\mathrm{Li}$ and Hong's [18], Sengar et al. [19], Hossain et al. [20], Gu et al. [3], and Pramunendar et al. [5]. Li and Hong [18] segmented and increased contrast in the image, then engineered features in the form of a combination of contour extraction and principal component analysis (PCA). Besides, a combination of Fisher and Mahalanobis distance models was used in the classification process [18]. Sengar et al. [19] modified the color space of the image and segmented the image processing stage. Identification was made manually based on three ranges of freshness that were determined by experts. However, their research used a dataset that was not interrupted by the background in the underwater environment. 
Meanwhile, several studies using a fish dataset from the marine environment were conducted by Hossain et al. [20], Gu et al. [3], and Pramunendar et al. [5]. Hossain et al. [20] proposed automatic ocean identification for video datasets using background reduction methods to detect moving objects, pyramid histograms of visual words (PHOW), and support vector machine (SVM). Gu et al. [3] applied the DCP technique to improve image quality and the superpixel aggregation technique for segmenting images, while template matching based on innerdistance shape context (IDSC) was used as the classification technique. Sun et al. [21] used an underwater dataset to improve image resolution using super-resolution. CNN and SVM methods were used to get features and classify them. The performance results given in this study amounted to $77.27 \%$. Rathi et al. [22] proposed CNN for classification technique; however, the image processing is used to enhance the segmentation process without enhancing the image quality. Pramunendar et al. [5] identified 23 fish species based on texture features and BPNN classification methods resulting in an accuracy of $93.37 \%$, which was applied to data that had been improved using contrast-adaptive color correction technique (NCACC).

From several previous studies, besides Pramunendar et al. [5], there are only a few that describe the relationship between improved image quality performance and classification performance of improved data. Most image processing techniques are used to separate objects and background images; the background image is arranged in such a way as to get fish objects quickly. On the other hand, several other researchers did not apply image processing in their research. Some studies only focus on the feature extraction method, classification method, or dataset used.

\section{The proposed approach}

Several studies have shown that the performance of classification methods is influenced by data, features, and classification methods. The performance of the classification method shows that the color, shape, and texture features do not always lead to poor accuracy. These features can be used to display image details precisely based on the functions completed. However, these features are influenced by data sources. To address this gap, this study presented a new workflow for identifying fish species by improving the quality of data sources (see Fig. 1). As shown in Fig. 1, the CLAHE image quality improvement method was implemented based on the

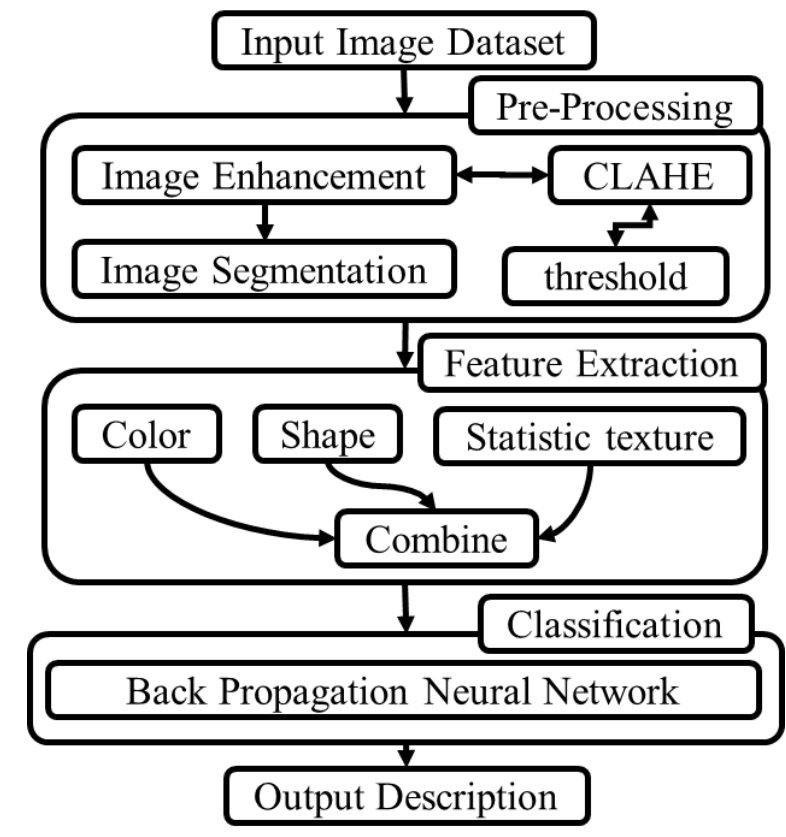

Figure. 1 The proposed workflow of fish species identification

appropriate threshold value to improve the quality of image data [10]. Then, images were converted into useful features by using a combination of feature methods. The results of those features were classified using the BPNN classification method [5].

\subsection{Image dataset}

This study assumed that sea conditions in Indonesia are similar to other locations in the world where objects are located in low-light environments. Therefore, the underwater image dataset used was obtained from LifeCLEF 2014 or the Fish4Knowlege dataset [23], which was downloaded at http://groups.inf.ed.ac.uk/f4k/. The Fish4Knowlege dataset consists of 23 fish species with a total of 27,370 fish images.

\subsection{Image processing stage}

The initial processing stage used in this approach model was a combination of dark channel prior (DCP) [6], and contrast limited adaptive histogram equalization (CLAHE) method [10], which was used to improve the quality of underwater images that are prone to bright color distortion and various noise that afflicts underwater objects. The stages of this technique are as follows:

- Estimating the transmission map by applying the lowest and highest light intensity in the local area from the effects of fog on underwater images.

- Applying filtering techniques and soft image mapping techniques to improve transmission maps for white balance problems. 
- Updating radians after the recovery process took place.

- Estimating ambient light based on projected transmissions.

- Implementing the CLAHE technique based on distribution parameters and clip limit parameters.

The distribution parameters in this study were uniform, rayleigh, and exponential [10]. Meanwhile, the clip limit parameter on CLAHE was done by using the value obtained from the FCM method as the threshold value applied to each image [24]. The best quality image results obtained were segmented manually based on the ground truth model provided in a public dataset to produce fish images without a background. This process was completed for each step of image processing, and all of them were applied and tested in the classification stage.

\subsection{Feature extraction stage}

\subsubsection{Color feature extraction}

The fish image used was in RGB color mode. Four RGB color features were extracted to classify fish species. The four-color features were mean, standard deviation, skewness, and kurtosis. The four features were obtained by using $P$ to initialize $I_{R}, I_{G}, I_{B}$, and $I_{R G B}$ which consists of red intensity, green intensity, blue intensity, and average intensity in RGB space. $M N$ refers to the number of pixels counted in fish images.

Feature extraction based on color consisted of average intensity, standard deviation, skewness, and kurtosis. The average intensity gave a measure regarding the distribution of colors. The mean intensity is expressed in $\mu_{R G B}$ and shown in Eq. (1).

$$
\mu_{R G B}=\frac{1}{M N} \sum_{k=1}^{M} \sum_{l=1}^{N} P_{k l}
$$

Standard deviations and variants were tools to determine the diversity of a group of data or the wide distribution of data distribution. The standard deviation expressed in $\sigma_{R G B}$ is shown in Eq. (2).

$$
\sigma_{R G B}=\sqrt{\frac{1}{M N} \sum_{k=1}^{M} \sum_{l=1}^{N}\left(P_{k l}-\mu_{R G B}\right)^{2}}
$$

Skewness ( $\left.\theta_{R G B}\right)$ states the measure of asymmetry. The distribution is said to be leaning to the left if it has a skewness value in the form of a negative number. In contrast, the distribution is said to be leaning to the right if it has a skewness value in the form of a positive number. If the distribution is symmetrical, the skewness coefficient is zero, as shown in Eq. (3).

$$
\theta_{R G B}=\frac{\frac{1}{M N} \sum_{k=1}^{M} \sum_{l=1}^{N}\left(P_{k l}-\mu_{R G B}\right)^{3}}{M N \sigma_{R G B}^{3}}
$$

Kurtosis $(\gamma)$ is a measure that shows the distribution of data that is tapered or dull (as shown in Eq. (4)).

$$
\gamma=\frac{\sum_{k=1}^{M} \sum_{l=1}^{N}\left(P_{k l}-\mu_{R G B}\right)^{4}}{M N \sigma_{R G B}^{4}}-3
$$

\subsubsection{Statistical texture feature extraction}

Two different types of statistical texture features were extracted in this study. One was a grayscale histogram-based texture, and the other was a graylevel texture (GLCM). Grayscale histogram stored the histogram brightness attribute statistics in an image. This study extracted general descriptors of grayscale histogram-based texture features of fish skin images, including mean intensity $(m)$, mean contrast $(\sigma)$, roughness $(R)$, third-order moment $\left(\mu_{3}\right)$, consistency $(U)$, and entropy $(e)$. The grayscale histogram-based texture features are described in Eq. (5) to (10), where $k$ is the gray level random of variable $i, L$ is the largest gray level in fish skin image, and $i$ is worth $0,1,2 \ldots, \mathrm{L}-1$. The probability of $k$ in the image is denoted by $p(k)$.

$$
\begin{aligned}
& m=\sum_{i=0}^{L-1} k p(k) \\
& \sigma=\sqrt{\sum_{i=0}^{L-1}(k-m)^{2} p(k)} \\
& R=1-\frac{1}{1+\sigma^{2}} \\
& \mu_{3}=\sum_{i=0}^{L-1}(k-m)^{3} p(k) \\
& U=\sum_{i=0}^{L-1} p^{2} k \\
& e=-\sum_{i=0}^{L-1} p(k) \log _{2} p(k)
\end{aligned}
$$

GLCM stores the probability of co-occurrence between two gray levels [25]. Special features of the fish skin image were extracted to describe the cooccurrence matrices data displayed in Eq. (11) to (15) and consisted of second-order moments or energy (ene), entropy (ent), contrast (con), homogeneity (hom), and correlation (cor). The distribution of cooccurrence values is represented as $k$ and $l$ at the offset given $(1,1)$ by $p(k, l)$, with different angles, $0^{0}$, $45^{\circ}, 90^{\circ}$, and $135^{\circ}$. Different angles were used so that the invariant rotation, mean, and the variance of the 
features that depended on the orientation was determined separately for different angles.

$$
\begin{aligned}
& \text { ene }=\sum_{k=0}^{L-1} \sum_{l=0}^{L-1}\{P(k, l)\}^{2} \\
& \text { ent }=-\sum_{k=0}^{G-1} \sum_{l=0}^{G-1} P(k, l) \times \log (P(k, l)) \\
& \text { con }=\sum_{n=0}^{G-1} n^{2}\left\{\sum_{k=0}^{G} \sum_{l=0}^{G} P(k, l)\right\}\{n=|k-l| \\
& \text { hom }=\sum_{k=0}^{G-1} \sum_{l=0}^{G-1} \frac{1}{1+(k-l)^{2}} P(k, l) \\
& \text { cor }=\frac{\sum_{k=0}^{G-1} \sum_{l=0}^{G-1}(k, l)\left(P(k, l)-\mu_{k}{ }^{\prime} \mu_{l}{ }^{\prime}\right.}{\sigma_{k}{ }^{\prime} \sigma_{l}{ }^{\prime}}
\end{aligned}
$$

The correlation is obtained using Eq. (16).

$$
\begin{aligned}
& P_{x}(k)=\sum_{l=0}^{G-1} P(k, l) \\
& P_{y}(l)=\sum_{k=0}^{G-1} P(k, l) \\
& \mu_{k}^{\prime}=\sum_{k=0}^{G-1} \sum_{l=0}^{G-1} k \times P(k, l) \\
& \mu_{l}^{\prime}=\sum_{k=0}^{G-1} \sum_{l=0}^{G-1} l \times P(k, l) \\
& \sigma_{k}^{\prime}=\sum_{k=0}^{G-1} \sum_{l=0}^{G-1} P(k, l)\left(k-\mu_{l}^{\prime}\right)^{2} \\
& \sigma_{l}^{\prime}=\sum_{k=0}^{G-1} \sum_{l=0}^{G-1} P(k, l)\left(l-\mu_{l}^{\prime}\right)^{2}
\end{aligned}
$$

\subsection{Classification stage}

The back-propagation propagation network (BPNN) method was used to test the results of image improvement by classifying each image consisting of various types of fish based on the Fish4Knowledge dataset. BPNN is a supervised learning algorithm, which consists of the training phase and the testing phase.

This method is divided into three layers, namely the input layer, the hidden layer, and the output layer. An input layer is capable of receiving data with multivariate types obtained from the feature extraction process in the form of a matrix with size $N$ data times $m$ features. The matrix is processed in a hidden layer using Eq. (17), with activation of the sigmoid function. The number of neurons is obtained from the average total attributes and the number of classes plus one. The hidden layer calculation results are the final result in the output layer, according to Eqs. (18) and (19). The output layer results are compared with the original label, resulting in a matching result that indicates the level of accuracy or the goal value of the BPNN method. The level of accuracy is also influenced by several parameters such as layer number, weight value, bias value, learning rate, momentum value, training cycle, and fault function. All parameters affect the error rate in the classification.

In the process, the level of misclassification was able to update each weight. Prediction errors were obtained by Eqs. (20) and (21), while weights were updated using Eqs. (22) and (23). The BPNN method stopped if the repetition was done according to the number of training cycles, as well as the level of error or expected goals.

$$
\begin{aligned}
& z=b_{i}+\sum_{i=0}^{n} x_{i} v_{i j} \\
& y=b_{0}+\sum_{i=0}^{n} x_{i} w_{i j} \\
& \delta_{y}=\left(t_{k}-z_{k}\right) f^{\prime}(y) \\
& \Delta v=\sum_{j=1}^{m} \delta_{y} w_{i j} \\
& \Delta w=a \delta_{y} z \\
& w_{\text {new }}=w_{\text {old }}+\Delta w \\
& v_{\text {new }}=v_{\text {old }}+\Delta v
\end{aligned}
$$

In Eqs. (17) to (23), $x$ is an input for $z$ in the form of multivariate data, while $z$ is a hidden layer obtained from the input process that is used as an input to get results. Meanwhile, $b$ is the value of bias, $v$ and $w$ are the input weights in the hidden layer, then $\alpha$ and $\delta$ are the learning rate and the result of prediction errors obtained from the difference between $y$ and $z$.

\subsection{Performance evaluation stage}

Performance evaluation of the classification method was done by calculating accuracy. Accuracy is defined as the correct classification of all data obtained. The accuracy value was obtained by using Eq. (8) with $t$ as the number of sample data classified correctly by experts, and $n$ as the total number of sample data.

$$
\text { accuracy }=\frac{t}{n} \times 100
$$

\section{Experiment design}

This study shows that the data source is essential to get the best accuracy. The technique applied to the 
fish4knowledge dataset was tested in the classification process so that the accuracy of the classification results was based on improved data. To observe the best accuracy, the accuracy obtained in this study was compared with previous studies [5].

The research process applied several parameters in each method used, such as distribution parameters and clip limit parameters. The determination of the clip limit parameter value was done by using the FCM method as the threshold value [24]. This parameter is not applied in several previous studies $[5,8]$. The best results from these parameters were used as default values in the process of setting the proposed method, while the parameters for some previous studies used default values from previous studies [1, 5]. Enhanced image quality was transformed into color-based and texture-based features. Color-based feature extraction methods used statistics from color pixels based on Eq. (1) to (4), and texture feature extraction used the histogram method in Eq. (5) and the GLCM method in Eq. (6). The total features generated were 120 features for each image. The color features generated 16 different features, while the texture features generated 104 features consisting of 24 histogram features and 80 GLCM features. These features were obtained based on each color layer. The color layers used were red, green, blue, and grayscale. In color-based features, each color layer generated four features, while texture-based features generated six features and 20 features for each color in the histogram and GLCMbased methods. Four features generated from color features were obtained based on Eqs. (1) to (4). Meanwhile, six features in the histogram method were obtained from Eqs. (5) to (10), and 20 features in the GLCM method were obtained based on four different angles with each corner producing five different features according to Eqs. (10) to (15). Therefore, features generated reached a total of 27,370 images multiplied by 120 features. The feature data was processed using the BPNN classification method.

Performance evaluation used sampling and validation methods with a value of 10 . The dataset was divided into ten values for cross-validation so that each section was distributed equally [5]. The experiment was repeated ten times according to the number of cross-validations, and the average results were obtained from the training process and performance classification testing. Performance evaluation was achieved after a confusion matrix was generated for the classification model. This study used MATLAB (www.mathworks.com) to enhance images, feature extraction, and classification of fish species.

\section{Results and discussion}

The discussion is divided into three stages consisting of the pre-processing stage, the feature extraction stage, and the classification stage (as shown in Fig. 2). Outputs are displayed at each stage of the study.

\subsection{Pre-processing image}

Enhanced and segmented images show that the CLAHE method can improve image quality. Results in Table 1 display quality improvements using the CLAHE method based on the rayleigh, uniform, and exponential distribution method. In addition to the distribution parameters, the clip limit parameter in this study used a default value of 0.02 . These results show that CLAHE can increase the contrast in the image adaptively so that it shows differences between objects.

The threshold value obtained by the FCM method was 0.5118 for the original image shown in Table 2 . The threshold method using FCM was used in the clip limit parameter to be able to produce images with excessive contrast. Larger clip limit improved the image quality by creating excessive contrast on the image.

Table 1. Pre-processing image using CLAHE

\begin{tabular}{|c|c|c|}
\hline $\begin{array}{c}\text { Enhance } \\
\text { technique }\end{array}$ & $\begin{array}{c}\text { Image } \\
\text { Fish_01 }\end{array}$ & $\begin{array}{c}\text { Segmented } \\
\text { Image }\end{array}$ \\
\hline ORIGINAL & & \\
\hline $\begin{array}{l}\text { CLAHE } \\
\text { rayleigh }\end{array}$ \\
CLAHE
\end{tabular}


Table 2. Pre-processing image using CLAHE with clip limit based on FCM threshold

\begin{tabular}{|c|c|c|}
\hline $\begin{array}{l}\text { Enhance } \\
\text { technique }\end{array}$ & $\begin{array}{c}\text { ClipLimit } \\
\text { default }\end{array}$ & $\begin{array}{l}\text { ClipLimit } \\
\text { FCM }\end{array}$ \\
\hline $\begin{array}{l}\text { CLAHE } \\
\text { rayleigh }\end{array}$ & & \\
\hline $\begin{array}{l}\text { CLAHE } \\
\text { uniform }\end{array}$ & & \\
\hline $\begin{array}{l}\text { CLAHE } \\
\text { exponential }\end{array}$ & & \\
\hline
\end{tabular}

Table 3. Feature extraction applied to original data without enhancement

\begin{tabular}{ccccccc}
\hline \hline No & X1 & X2 & X3 &. & X120 \\
\hline \hline 1 & 0.728 & 297.408 & 0.870 &. &. & 7.314 \\
\hline 2 & 0.735 & 310.698 & 0.873 &. &. & 6.224 \\
\hline 3 & 0.728 & 270.399 & 0.872 &. &. & 7.970 \\
\hline. &. &. &. &. &. &. \\
\hline. &. &. &. &. &. &. \\
\hline 27,369 & 0.834 & 224.621 & 0.934 &. &. & 8.147 \\
\hline 27,370 & 0.807 & 267.166 & 0.923 &. &. & 6.311 \\
\hline
\end{tabular}

\subsection{Feature extraction}

The improved image quality was transformed into a useful feature. The combination of feature methods generated a new matrix with a size of 27,370 images $\times 120$ features. Each row represented data, and the matrix column stated the features of each information.

As shown in Table 3, some columns contain variables $X 1, X 2$, to $X 120$. The column is a variable containing each value based on texture and color features. $X 1, X 2$, and $X 3$ are the second-moment angle features, contrast, and correlation of the GLCM feature method with orientation direction 0 degrees on the red layer. Meanwhile, other $X n$ features were a combination of orientation and color layers in the GLCM method and the histogram feature and color feature method. Furthermore, the X120 feature was the kurtosis value in the gray layer. The matrices in rows 1 through 27,370 were the data numbers obtained from the dataset. In this study, all features were used in the classification process; thus, it was assumed that all features contributed to getting the best classification performance.

\subsection{Evaluation using several classification methods}

The results of the obtained features were a combination of color features and texture features. The next step was to get the accuracy value in the data. This study used several classification methods to evaluate the features extracted from image enhancements and compared results to original image data that was not enhanced. The classification methods used include support vector machine (SVM) $[14,21]$, linear discriminant analysis (LDA) [15], Naïve Bayes (NB) [12], k-nearest neighbor (KNN) [16], and decision tree (DT) [17].

The parameters for the experiment were determined based on the default values from the study [5], with the following details: (1) The best settings for Operator type C-SVC SVM methods ranged from 0.1 to $0.9,1$ to 9 , and 10 to 100 with RBF kernel; (2) The KNN method used $\mathrm{k}$ parameter values ranging from 1 to 23 according to the whole class in the data and some distance methods. The best accuracy performance was obtained at nine at $k$ value and the maxproductsimilarity distance method. (3) The parameter setting for the DT method was obtained from the ratio for separation, with minimum split sizes and minimum leaf notes of 4 and 2, respectively. The confidence level was $9.5 \%$, and the increase was at least 0.1 . Meanwhile, (4) the setting parameters for the BPNN method ranged from 0.1 to 1 for momentum and learning levels, but the training cycle was configured to be 1000 cycles. The best parameter values for momentum, learning, and training levels were $0.1,0.1$, and 1000 , respectively. In addition, the 53 hidden layers were used in this study.

Table 4 shows that improving images using the CLAHE method increases the accuracy of each classification method. The results were obtained with the RGB color space applied to the CLAHE parameter in the form of a 'uniform' distribution and

Table 4. Accuracy of several classification methods based on the CLAHE enhancement technique and combination features

\begin{tabular}{cccc}
\hline \hline Method & Original & Enhanced & increase \\
\hline \hline BPNN & 93.10 & 94.81 & 1.71 \\
\hline DT & 81.70 & 82.45 & 0.75 \\
\hline KNN & 84.00 & 87.14 & 3.14 \\
\hline LDA & 90.79 & 92.71 & 1.92 \\
\hline NB & 33.90 & 37.01 & 3.11 \\
\hline SVM & 79.98 & 86.14 & 6.16 \\
\hline
\end{tabular}


a clip limit value of 0.02 , as well as a combination of texture and color-based feature extraction methods. The highest performance accuracy for classification using the CLAHE method was obtained using the BPNN method. However, the SVM method shows the highest performance increase, followed by the KNN and NB method, which were $6.16 \%, 3.14 \%$, and $3.11 \%$, respectively. Meanwhile, the BPNN method only obtained a performance increase of $1.71 \%$ to reach $94.81 \%$. The highest performance of BPNN is influenced by the fact that it is more flexible in handling data that has a long and full feature size. Meanwhile, other classification methods also have advantages, but they are not as flexible as BPNN in handling significant features.

\subsection{Evaluation of CLAHE distribution based on colorspace using BPNN classification method}

The improved images were tested by the BPNN classification method. This experiment used several parameters of the CLAHE method extracted using the GLCM feature extraction combination method and was classified by the BPNN classification method, where the BPNN parameters used the results of parameters from previous experiments [5]. Some of these parameters included the stratified sampling method with 10-fold cross-validation, the learning rate of 0.1 , the momentum of 0.1 , and the training cycle of 1000 cycles.

Table 5 shows that the CLAHE method with a uniform distribution provided the highest performance. This could be caused by the fact that the flat data distribution given matched the image being tested. It is possible that the Rayleigh distribution got under uniform distribution because of the size of the image in the data or the size of the vector generated from the data. The small data size allowed the Rayleigh distribution to tend not to be able to distribute more in the middle gray value, and the lower frequencies cannot be well dispersed by the exponential distribution.

Table 5. Classification accuracy of CLAHE distribution based on combination feature and BPNN classification

\begin{tabular}{cccc}
\hline \hline Method & uniform & rayleigh & exponential \\
\hline \hline RGB & 94.812 & 92.656 & 92.072 \\
\hline HSV & 94.812 & 94.154 & 92.985 \\
\hline LAB & 54.439 & 54.330 & 53.526 \\
\hline LIN & 95.579 & 94.812 & 93.460 \\
\hline XYZ & 94.446 & 93.022 & 91.268 \\
\hline YCBCR & 93.752 & 89.916 & 89.770 \\
\hline \hline
\end{tabular}

\subsection{Evaluation of CLAHE distribusion based on colorspace using BPNN classification method}

At this stage, the performance of the BPNN method was applied to each color space before the CLAHE color improvement was performed. The compared color spaces based on Gonzalez [26], were RGB (red, green and blue), HSV (hue, saturation, and value), LAB (linearized sRGB), LIN (Linearize gamma-corrected RGB), XYZ (linearized sRGB), and $\mathrm{YCbCr}$ (luminance).

The RGB color space represents images in numbers whose elements determine the intensity values of the red, green, and blue color layers. HSV color space (hue, saturation, value) is an alternative to the RGB color model, to harmonize human vision in viewing the attributes of color making. The colors of each HSV layer are arranged in radial slices, around the central axis of a neutral color that ranges from black on the bottom to white on the top. The $\mathrm{XYZ}$ color space is a development of the CIE color space. The $Y$ layer represents the illumination of color, and the $\mathrm{Z}$ layer is related to the amount of blue in the image, but the $\mathrm{Z}$ value is not identical to the $\mathrm{B}$ value in the RGB color space while the $X$ layer does not have a clear color analogy. The color space $\mathrm{L}^{*} \mathrm{a}^{*} \mathrm{~b}^{*}$ shows uniformity between color spaces perceptually rather than XYZ color space. The color in the $\mathrm{L}^{*} \mathrm{a} * \mathrm{~b}^{*}$ color space is not part of the RGB color space. Meanwhile, the LIN color space applied RGB color modification based on gamma correction.

The results of the six color spaces were inputted to the CLAHE method, and a combination of feature extraction was applied for the classification process using the BPNN classification method. The experimental results between the color space comparison and the distribution method are shown in Table 5. The best color space results in this application were obtained using the LIN color space. This could be caused by the fact the distribution of the given data in accordance with the image being tested was done by gamma correction to correct the difference between the way the camera captured the content, and the way the content was displayed against how the eye responds to light. Similar to LIN, HVS has the ability to respond nonlinearly to light levels, making it more sensitive to small changes in the dark area. However, HSV is not sensitive in bright areas. The difference between LIN and HSV lies in need of coding allocation. HSV needs coding allocation of 11 bit, while LIN is around 8 bits [27]. 
Table 6. Comparison of BPNN performance based on extraction feature

\begin{tabular}{lcccccc}
\hline \hline & GLCM & Proposed & LBP & SFTA & STC & STT \\
\hline \hline HSV EXPONENTIAL & 89.478 & 92.985 & 88.601 & 47.351 & 79.357 & 87.797 \\
\hline HSV RAYLEIGH & 90.354 & 94.154 & 89.624 & 44.355 & 84.107 & 89.039 \\
\hline HSV UNIFORM & 91.341 & 94.812 & 90.976 & 44.282 & 85.130 & 90.026 \\
\hline LAB EXPONENTIAL & 53.708 & 53.526 & 75.374 & 45.342 & 49.945 & 73.109 \\
\hline LAB RAYLEIGH & 53.745 & 54.330 & 77.932 & 44.282 & 49.982 & 72.963 \\
\hline LAB UNIFORM & 54.512 & 54.439 & 79.211 & 44.794 & 49.982 & 74.863 \\
\hline LIN EXPONENTIAL & 89.551 & 93.460 & 89.222 & 87.468 & 82.134 & 89.806 \\
\hline LIN RAYLEIGH & 91.195 & 94.812 & 91.524 & 62.952 & 82.463 & 90.793 \\
\hline LIN UNIFORM & 92.364 & 95.579 & 92.802 & 79.503 & 82.463 & 90.829 \\
\hline RGB EXPONENTIAL & 88.272 & 92.072 & 88.783 & 88.820 & 83.449 & 89.478 \\
\hline RGB RAYLEIGH & 89.660 & 92.656 & 89.916 & 69.309 & 82.864 & 91.341 \\
\hline RGB UNIFORM & 92.912 & 94.812 & 91.195 & 82.243 & 84.691 & 92.035 \\
\hline XYZ EXPONENTIAL & 88.418 & 91.268 & 87.614 & 88.089 & 79.247 & 89.258 \\
\hline XYZ RAYLEIGH & 90.464 & 93.022 & 89.953 & 68.615 & 83.887 & 89.076 \\
\hline XYZ UNIFORM & 91.487 & 94.446 & 91.524 & 80.088 & 83.851 & 89.368 \\
\hline YCBCR EXPONENTIAL & 87.980 & 89.770 & 88.126 & 44.720 & 76.434 & 82.718 \\
\hline YCBCR RAYLEIGH & 88.710 & 89.916 & 88.929 & 44.465 & 82.682 & 85.970 \\
\hline YCBCR UNIFORM & 91.341 & 93.752 & 89.149 & 44.282 & 82.828 & 85.020 \\
\hline
\end{tabular}

\subsection{Evaluation of CLAHE distribusion based on colorspace using BPNN classification method}

At this stage, the performance of the BPNN method was applied to several feature extraction methods after the CLAHE color improvement. The CLAHE color improvement method was based on the distribution parameters of the CLAHE, namely exponential (exp), Rayleigh (ray), and uniform (uni). Some feature extraction methods used in this study include gray-level co-occurrence matrix (GLCM) [25], local binary pattern (LBP) [28], segmentationbased fractal texture analysis (SFTA) [29], histogram based on color (STC) [30], and histogram based on texture (STT) [30].

Table 6 shows that the proposed method obtained the highest performance. The method was a combination of features that used the LIN color space. The use of a combination of color and texture feature extraction methods obtained the top 10 highest accuracy values from the experiments conducted. The combination of feature extraction used consisted of the method of feature extraction of gray level cooccurrence matrix (GLCM), histogram based on texture (STT), and histogram based on color (STC). This could be caused by the fact that underwater image data has high color dominance; thus, it allows texture-based features to not get incomplete
Table 7. Comparison of BPNN accuracy between the GLCM method [5] and the proposed method

\begin{tabular}{cccc} 
& GLCM & Proposed & increase \\
\cline { 2 - 4 } ORIGINAL & $89.05 \%$ & $93.10 \%$ & $4.05 \%$ \\
\hline CLAHE & $91.25 \%$ & $94.81 \%$ & $3.56 \%$ \\
\hline NCACC & $93.73 \%$ & $96.05 \%$ & $2.32 \%$ \\
\hline
\end{tabular}

information. Therefore, a combination of several features is needed to get high accuracy performance.

Table 6 shows that the proposed method obtained the highest performance. The method was a combination of features that used the LIN color space. The use of a combination of color and texture feature extraction methods obtained the top 10 highest accuracy values from the experiments conducted. The combination of feature extraction used consisted of the method of feature extraction of gray level cooccurrence matrix (GLCM), histogram based on texture (STT), and histogram based on color (STC). This could be caused by the fact that underwater image data has high color dominance; thus, it allows texture-based features to not get incomplete information. Therefore, a combination of several features is needed to get high accuracy performance.

Table 7 shows the results of a comparison between the workflow of previous studies [5] with the workflow of this study. Accuracy of results on data extracted with a combination of the proposed methods provided performance improvements of 
Compared to the proposal in previous studies [5] the proposed method in the form of a combination of methods could increase the accuracy by $2.32 \%$. When the proposed combination of features was applied to the original data, the accuracy increased by $4.05 \%$ compared to previous features results. In addition, when the proposed combination of features was applied to the CLAHE image enhancement method, the increase was $3.56 \%$. The accuracy performance could be caused by the fact that competent features obtained from a combination of color and texture feature generated better resulting features.

\section{Conclusions}

This study was an effort to identify various types of endangered aquatic biota, especially fish. Therefore, it could solve one of the most difficult challenges faced by many countries. Our previous research proposed automatic fish identification techniques by considering problems in the original data with data taken from various environmental conditions. This study presented a new workflow in identifying fish species, which was expected to provide a high level of accuracy when the proposed method was applied in classifying fish images underwater. The proposed model improved the accuracy by $3.56 \%$, implied in the data of 27,370 images consisting of 23 species of fish using the proposed method in the form of improved CLAHE image quality with uniform distribution and clip limit parameters by 0.02 , and extracted using a combination of color features and texture. These results indicate that the proposed method improves accuracy by improving the quality of the original image underwater. However, the increase in this study was smaller than the increase in accuracy obtained from previous studies if the proposed method in the form of improving the quality of NCACC images was applied to the feature combination method in this study. Improved accuracy was obtained because the characteristics of objects were getting better, and the differences between objects in the picture could be more apparent. The results show that the image quality improvement method using CLAHE was able to improve the accuracy of all the classification methods tested, and the combination feature extraction method was also able to improve accuracy. Therefore, it can be concluded that the method of image enhancement and feature extraction methods affect the accuracy of the identification of fish species. Hence, improvement at each processing stage is essential to show the characteristics of each image and further improve the quality of classification performance.

\section{Conflicts of Interest}

In accordance with the International Journal of Intelligent Engineering and Systems, policy, and my ethical obligation as a researcher, I am reporting that I have received a research grant from the Indonesian Ministry of Research and Higher Learning (DPRMDIKTI). I have disclosed those interests fully to the International Journal of Intelligent Engineering and Systems, and I have in place an approved plan for managing any potential conflicts arising from those research grants.

\section{Author Contributions}

This research articles involve several authors and contributions every author is provided in a short paragraph. The conceptualization about this topic and the methodology is defined by Ricardus Anggi Pramunendar; application of software by Dwi Puji Prabowo; validation about data and result by Pulung Nurtantio Andono, and Moch Arief Soeleman; the formal analysis by Ricardus Anggi Pramunendar; investigation about resources and curation of data by Dwi Puji Prabowo; for the writing process - the original draft preparation and the visualization by Ricardus Anggi Pramunendar; review and editing is defined by Yuslena Sari; supervision, Pulung Nurtantio Andono; project administration, Dewi Pergiwati; source of funding by Indonesian Ministry of Research and Higher Learning (DPRM-DIKTI).

\section{Acknowledgments}

This work funded by Indonesian Ministry of Research and Higher Learning (DPRM-DIKTI) including supported by the Faculty of Computer Science, Universitas Dian Nuswantoro.

\section{References}

[1] R. A. Pramunendar, S. Wibirama, and P. I. Santosa, "A Novel Approach for Underwater Image Enhancement based on Improved Dark Channel Prior with Colour Correction", Journal of Engineering Science and Technology, Vol. 13, No. 10, pp. 3220-3237, 2018.

[2] M. Suganuma, D. Tsuchiya, S. Shirakawa, and T. Nagao, "Hierarchical Feature Construction for Image Classification using Genetic Programming", In: Proc. of IEEE International Conf. on Systems, Man, and Cybernetics, pp. 001423-001428, 2016.

[3] Z. Gu, R. Wang, J. Dai, H. Zheng, and B. Zheng, 
"Automatic Searching of Fish from Underwater Images via Shape Matching", In: Proc. of OCEANS, Shanghai, pp. 1-4, 2016.

[4] R. A. Pramunendar, G. F. Shidik, C. Supriyanto, P. N. Andono, and M. Hariadi, "Auto Level Color Correction for Underwater Image Matching Optimization", International Journal of Computer Science and Network Security, Vol. 13, No. 1, pp. 18-23, 2013.

[5] R. A. Pramunendar, S. Wibirama, P. I. Santosa, P. N. Andono, and M. A. Soeleman, "A Robust Image Enhancement Techniques for Underwater Fish Classification in Marine Environment", International Journal of Intelligent Engineering and Systems, Vol. 12, No. 5, pp. 116-239, 2019.

[6] N. Carlevaris-Bianco, A. Mohan, and R. M. Eustice, "Initial Results in Underwater Single Image Dehazing", In: Proc. of OCEANS MTS/IEEE, Seattle, pp. 1-8, 2010.

[7] H. Wen, Y. Tian, T. Huang, and W. Gao, "Single Underwater Image Enhancement with a New Optical Model", In: Proc. of IEEE International Symposium on Circuits and Systems, pp. 753756, 2013.

[8] E. H. Kaur and R. Mahajan, "Improved Dark Channel Prior Using Gabor Filter and Clahe", International Journal of Application or Innovation in Engineering \& Management, Vol. 3, No. 7, pp. 127-134, 2014.

[9] A. Galdran, D. Pardo, A. Picón, and A. AlvarezGila, "Automatic Red-Channel Underwater Image Restoration", Journal of Visual Communication and Image Representation, Vol. 26, pp. 132-145, 2015.

[10] Pujiono, N. A. Pulung, I. K. E. Purnama, and M. Hariadi, "Color Enhancement of Underwater Coral Reef Images using Contrast Limited Adaptive Histogram Equalization (CLAHE) with Rayleigh Distribution", In: Proc. of International Conf. on Information, Communication, Technology, and Systems, pp. 45-52, 2013.

[11]D. J. Lee, R. B. Schoenberger, D. Shiozawa, X. $\mathrm{Xu}$, and P. Zhan, "Contour Matching for a Fish Recognition and Migration-Monitoring System", In: Proc. of SPIE 5606, Two- and ThreeDimensional Vision Systems for Inspection, Control, and Metrology II, Vol. 5606, p. 37, 2004.

[12] M. S. Nery, A. M. Machado, M. F. M. Campos, F. L. C. Pádua, R. Carceroni, and J. P. QueirozNeto, "Determining the Appropriate Feature Set for Fish Classification Tasks", In: Proc. of Brazilian Symposium of Computer Graphic and Image Processing, Vol. 2005, pp. 173-180,
2005.

[13] D. J. White, C. Svellingen, and N. J. C. Strachan, "Automated measurement of Species and Length of Fish by Computer Vision", Fisheries Research, Vol. 80, No. 2-3, pp. 203-210, 2006.

[14] A. Rova, G. Mori, and L. M. Dill, "One Fish, Two Fish, Butterfish, Trumpeter: Recognizing Fish in Underwater Video", In: Proc. of Conference on Machine Vision Applications, pp. 404-407, 2007.

[15] R. Larsen, H. Olafsdottir, and B. K. Ersbøll, "Shape and Texture based Classification of Fish Species", Image Analysis, pp. 745-749, 2009.

[16] N. M. S. Iswari, Wella, and Ranny, "Fish Freshness Classification Method Based on Fish Image using k-Nearest Neighbor", In: Proc. of the 4th International Conference on New Media Studies, Vol. 2017, pp. 87-91, 2017.

[17] W. N. Khotimah, A. Z. Arifin, A. Yuniarti, A. Y. Wijaya, D. A. Navastara, and M. A. Kalbuadi, "Tuna Fish Classification using Decision Tree Algorithm and Image Processing Method", In: Proc. of International Conference on Computer, Control, Informatics and its Applications, pp. 126-131, 2015.

[18] L. Li and J. Hong, "Identification of Fish Species Based on Image Processing and Statistical Analysis Research", In: Proc. of IEEE International Conference on Mechatronics and Automation, pp. 1155-1160, 2014.

[19] N. Sengar, V. Gupta, M. K. Dutta, and C. M. Travieso, "Image Processing based Method for Identification of Fish Freshness using Skin Tissue", In: Proc. of the 4th International Conference on Computational Intelligence \& Communication Technology, No. Cict, pp. 1-4, 2018.

[20] E. Hossain, S. M. S. Alam, A. A. Ali, and M. A. Amin, "Fish Activity Tracking and Species Identification in Underwater Video", In: Proc. of the 5th International Conference on Informatics, Electronics and Vision, pp. 62-66, 2016.

[21] X. Sun, J. Shi, J. Dong, and X. Wang, "Fish Recognition from Low-Resolution Underwater Images", In: Proc. of the 9th International Congress on Image and Signal Processing, BioMedical Engineering and Informatics, pp. 471-476, 2016.

[22] D. Rathi, S. Jain, and S. Indu, "Underwater Fish Species Classification using Convolutional Neural Network and Deep Learning", In: Proc. of the 9th International Conference on Advances in Pattern Recognition, ICAPR 2017, pp. 344349, 2018.

[23] B. J. Boom, P. X. Huang, J. He, and R. B. Fisher, 
"Supporting Ground-Truth Annotation of Image Datasets using Clustering", In: Proc. of the 21st International Conference on Pattern Recognition, pp. 1542-1545, 2012.

[24] M. A. Soeleman, M. Hariadi, and M. H. Purnomo, "Adaptive Threshold for Background Subtraction in Moving Object Detection using Fuzzy C-Means Clustering”, In: Proc. of the TENCON IEEE Region 10 Conference, pp. 1-5, 2012.

[25] S. Santosa, R. A. Pramunendar, D. P. Prabowo, and Y. P. Santosa, "Wood Types Classification using Back-Propagation Neural Network based on Genetic Algorithm with Gray Level Cooccurrence Matrix for Features Extraction", IAENG International Journal of Computer Science, Vol. 46, No. 2, 2019.

[26] R. C. Gonzalez and R. E. Woods, Digital Image Processing, 2008.

[27] D. R. Bull, "Digital Picture Formats and Representations", Communicating Pictures, Elsevier, pp. 99-132, 2014.

[28] J. Kaewchote, S. Janyong, and W. Limprasert, "Image Recognition Method using Local Binary Pattern and the Random Forest Classifier to Count Post Larvae Shrimp", Agriculture and Natural Resources, Vol. 52, No. 4, pp. 371-376, 2018.

[29] A. F. Costa, G. Humpire-Mamani, and A. J. M. H. Traina, "An efficient Algorithm for Fractal Analysis of Textures", In: Proc. of the Brazilian Symposium of Computer Graphic and Image Processing, pp. 39-46, 2012.

[30] J. Hu, D. Li, Q. Duan, Y. Han, G. Chen, and X. $\mathrm{Si}$, "Fish Species Classification by Color, Texture and Multi-Class Support Vector Machine using Computer Vision", Computers and Electronics in Agriculture, Vol. 88, pp. 133-140, 2012. 